\title{
Organisation und Algorithmus
}

\section{Wie algorithmische Kategorien, Vergleiche und Bewertungen durch Organisationen relevant gemacht werden}

\author{
Stefanie Büchner • Henrik Dosdall
}

Eingegangen: 12. September 2020 / Angenommen: 3. Oktober 2020 / Online publiziert: 29. Juni 2021

(C) Der/die Autor(en) 2021

Zusammenfassung Der vorliegende Beitrag analysiert, wie Organisationen Algorithmen, die wir als digitale Beobachtungsformate verstehen, mit Handlungsfähigkeit ausstatten und damit actionable machen. Das zentrale Argument lautet, dass die soziale Relevanz digitaler Beobachtungsformate sich daraus ergibt, dass und wie sie in organisationale Entscheidungsarchitekturen eingebettet sind. Diesen Zusammenhang illustrieren wir am Beispiel des österreichischen Arbeitsmarktservice (AMS), der 2018 einen Algorithmus einführte, um die Integrationschancen arbeitsuchender Personen zu bewerten. Der AMS steht dabei stellvertretend für aktuelle Bestrebungen vieler Organisationen, algorithmische Systeme einzusetzen, um knappe öffentliche Ressourcen vermeintlich effizienter zu distribuieren. Um zu rekonstruieren, wie dies geschieht, zeigen wir, welche Operationen des Kategorisierens, Vergleichens und Bewertens das algorithmische Modell vollzieht. Darauf aufbauend demonstrieren wir, wie das algorithmische Modell in die organisationale Entscheidungsarchitektur eingebunden ist. Erst durch diese Einbindung - die Möglichkeit, Unterschiede für andere, relativ stabil erzeugte Entscheidungen zu machen - entfaltet das digitale Beobachtungsformat soziale Relevanz. Abschließend argumentieren wir, dass algorithmische Modelle, wie sie am Fall des AMS beobachtet werden können, dazu tendieren, sich in Organisationen zu stabilisieren. Dies begründen wir damit, dass die organisationalen Lernchancen im Umgang mit dem Algorithmus dadurch reduziert sind, dass dieser in einem Bereich zum Einsatz kommt, der durch Technologiedefizit und koproduktive Leistungserstellung geprägt ist.

\footnotetext{
S. Büchner $(\square)$

Institut für Soziologie, Leibniz Universität Hannover

Im Moore 21, 30167 Hannover, Deutschland

E-Mail: s.buechner@ish.uni-hannover.de

H. Dosdall

Wirtschafts- und Sozialwissenschaftliche Fakultät, Universität Potsdam

August-Bebel-Str. 89, 14482 Potsdam, Deutschland

E-Mail: henrik.dosdall@uni-potsdam.de
} 
Schlüsselwörter Digitalisierung · Öffentliche Organisationen · Algorithmen · Organisationales Lernen · Digitale Beobachtungsformate

\title{
Organization and Algorithm
}

How Organizations Make Algorithmic Categories, Comparisons, and Evaluations Relevant

\begin{abstract}
This article analyzes how organizations endow algorithms, which we understand as digital formats of observation, with agency, thus rendering them actionable. Our main argument is that the relevance of digital observation formats results from how organizations embed them in their decision architectures. We demonstrate this using the example of the Austrian Public Employment Service (AMS), which introduced an algorithm in 2018 to evaluate the chances of unemployed persons being reintegrated in the labor market. In this regard, the AMS algorithm serves as an exemplary case for the current trend among public organizations to harness algorithms for distributing limited resources in a purportedly more efficient way. To reconstruct how this is achieved, we delineate how the AMS algorithm categorizes, compares, and evaluates persons. Building on this, we demonstrate how the algorithmic model is integrated into the organizational decision architecture and thereby made actionable. In conclusion, algorithmic models like the AMS algorithm also pose a challenge for organizations because they mute chances for realizing organizational learning. We substantiate this argument with regard to the role of coproduction and the absence of clear causality in the field of (re)integrating unemployed persons in the labor market.
\end{abstract}

Keywords Digitization · Public organizations · Algorithms · Organizational learning $\cdot$ Digital observation formats

\section{Einleitung}

Vergleiche sind sozial folgenreiche Operationen. Mehr noch sind sie ,ein konstitutives Moment sozialer Ordnung" (Heintz 2010, S. 163). Dabei verstehen wir unter Vergleichen im Anschluss an Bettina Heintz $(2016,2019)$ sowie Léa Renard und Theresa Wobbe (2019) eine zweischrittige Operation, die zunächst voraussetzt, Vergleichbarkeit sozial herzustellen (Zerubavel 1996). Erst wenn diese postuliert ist (Renard und Wobbe 2019, S. 596), lassen sich Sachverhalte ,auf ihre Unterschiede hin [...] beobachten“ (Heintz 2016, S. 309). Die soziale Relevanz dieser Operationen zeigt sich dort, wo verglichene Personen und Sachverhalte kategorisiert und bewertet werden. Dies beeinflusst maßgeblich, wie Personen sich selbst wahrnehmen (Festinger 1954), sich öffentlich äußern (Noelle-Neumann 1974), wie sie entscheiden (Gigerenzer und Gaissmaier 2011) und welche Risiken sie einzugehen bereit sind (Kahneman und Tversky 2000).

Der vorliegende Beitrag schließt an diese Arbeiten an, indem er aus organisationssoziologischer Perspektive zeigt, dass algorithmische Prozesse des Vergleichens, Bewertens und Kategorisierens insbesondere dann sozial folgenreiche Operationen 
sind, wenn sie prominent in organisationale Entscheidungsarchitekturen eingebunden werden (vgl. Büchner 2018a). Dieses Argument werden wir am Fall des österreichischen Arbeitsmarktservice (AMS) illustrieren, der seit 2019 einen Algorithmus einführt (AMS-Algorithmus), um zu entscheiden, in welchem Umfang arbeitssuchenden Personen Unterstützungsleistungen gewährt werden. Der Algorithmus macht Arbeitssuchende zunächst mittels verschiedener Variablen vergleichbar, um sie anschließend hinsichtlich ihrer Integrationschancen zu bewerten und darauf basierend einer von drei Kategorien zuzuordnen. Die unterschiedliche Kategorisierung der Personen weist diesen Leistungen in unterschiedlichem Format und Umfang zu. Da der AMS-Algorithmus die Vergleichspraktiken auf Basis digitaler Technologien ins Werk setzt, verstehen wir im Anschluss an Bettina Heintz (2021, in dieser Ausgabe) den AMS-Algorithmus als ein digitales Beobachtungsformat. ${ }^{1}$ Das zentrale Argument der folgenden Ausführungen lautet daher, dass der Österreichische Arbeitsmarktservice dieses digitale Beobachtungsformat actionable macht, also mit Handlungsfähigkeit ausstattet, indem er den Algorithmus folgenreich in seine organisationale Entscheidungsarchitektur einbindet. Erst durch diese Einbindung kann der Algorithmus Unterschiede für andere, relativ stabil erzeugte Entscheidungen machen. Unser theoretischer Ausgangspunkt ist somit die Entscheidungsförmigkeit von Organisationen (March und Simon 1958; Luhmann 2006). Von hier aus zeigen wir, dass und wie die ,social power“ (Beer 2017) digitaler Beobachtungsformate dadurch hergestellt wird, dass sie auf spezifische Weise in organisationale Entscheidungsarchitekturen eingebettet werden.

Mit unserem Argumentationsgang folgen wir Arbeiten, die die zentrale gesellschaftliche Bedeutung von Organisationen betonen (Perrow 1991; Coleman 1974; Luhmann 2006). Wir begründen folglich, dass Beobachtungsformate, wie Hochschulrankings (Brankovic et al. 2018; Espeland und Sauder 2007), FICO Scores (Fourcade 2018), Ratings (Esposito und Stark 2019; Kette 2018; Strulik 2004) oder Risikoprämien von Kreditausfallversicherungen (Schwarting 2015; MacKenzie 2011), nicht aus sich heraus bedeutsam sind, sondern weil sie zur Grundlage organisierter Entscheidungen werden. Folgt man der von Maja Apelt und Veronika Tacke (2012) angeregten Unterscheidung verschiedener Organisationstypen, lässt sich zudem eine Besonderheit des AMS-Falls sichtbar machen, die die soziale Relevanz von Organisationen nochmals hervorhebt. Während Ratingagenturen, Zeitungen und Internetportale Rankings und Ratings potenziell exzessiv (Abbott 2014) anfertigen können, legen die Vergleichspraktiken öffentlicher Organisationen häufig fest, wie summenkonstante und begrenzte Ressourcen zu verteilen sind. Dies trifft auch auf den AMS-Algorithmus zu, der genau an einer solchen sozialpolitisch sensiblen Stelle ansetzt: der Verteilung begrenzter öffentlicher Ressourcen.

Im Fall des österreichischen Arbeitsmarktservice kreuzt sich damit die Problematik, wie begrenzte öffentliche Ressourcen zu verteilen sind mit dem Einsatz algorithmischer Technologien, um diesen Vorgang zu optimieren. Diese Charakteristika

\footnotetext{
$1 \mathrm{Zu}$ diesen digitalen Beobachtungsformaten zählen viele der in dieser Ausgabe analysierten Formate, wie z. B. Recommendersysteme (Heintz und Unternäherer 2021, in dieser Ausgabe) und Dating-Apps (Peetz 2021, in dieser Ausgabe), aber auch Algorithmen zur prädiktiven polizeilichen Ermittlung (Brayne 2021, in dieser Ausgabe) und Diagnose (Maiers 2017).
} 
des AMS-Falles wiederum ermöglichen es, zu analysieren, wie sich digitale Technologien und kritische Bereiche organisierten Entscheidens gegenseitig durchdringen (Büchner 2018a; Kette 2020), was unterstreicht, dass die soziale Relevanz organisierter Vergleichspraktiken durch die Digitalisierung gesteigert wird (Danaher 2016; Beer 2017).

Die dargelegte organisationssoziologische Perspektive ergänzt den analytischen Fokus des Bandes auf Vergleichspraktiken, indem wir zeigen, wie Vergleiche, Bewertungen und Kategorisierungen digitalisierter Beobachtungsformate durch ihre organisationale Einbettung actionable gemacht werden. Dazu beschreiben wir in einem ersten Schritt den AMS-Algorithmus und erläutern, wie der Algorithmus vergleicht, bewertet und kategorisiert (Abschn. 2). Danach illustrieren wir, wie der AMS-Algorithmus in die Entscheidungsarchitektur des AMS eingebettet ist und dadurch actionable gemacht wird (Abschn. 3). Dieses Argument kontextualisieren wir, indem wir darlegen, dass Organisationen Algorithmen auf unterschiedliche Art und Weise in Entscheidungsarchitekturen einbinden. Darauf aufbauend synthetisieren wir unsere vorherigen Analyseschritte, indem wir fragen, welche Lernchancen Algorithmen, wie der AMS-Algorithmus, bieten (Abschn. 4). Abschließend fassen wir unsere Ausführungen zusammen (Abschn. 5).

\section{Fallbeschreibung und methodische Vorbemerkung}

Algorithmen operieren nie als reiner Vollzug mathematischer Modelle, sondern sind immer eingebunden in soziotechnische Arrangements. Sie operieren also ,,in collaboration with data, technologies, people, [...] under varying conditions“. Infolgedessen entfalten sie ihre Effekte, in contingent and relational ways, producing localised and situated outcomes“ (Kitchin 2017, S. 25). Hinzu kommt, dass Algorithmen üblicherweise Teil ,,algorithmischer Systeme“ sind (Seaver 2017; Munn 2018). So arbeitet auch der AMS-Algorithmus mit mehr als 90 Modellen gleichzeitig (Kienzl und Szigetvari 2018). Weil uns jedoch im Folgenden ein bestimmtes Verhältnis - das zwischen organisierten Entscheidungsarchitekturen und Algorithmen - interessiert, verwenden wir eine hierauf fokussierte Definition von Algorithmen. Algorithmen verstehen wir im Folgenden als Regeln, die mittels vorab definierter Schritte und Modelle einen Output erzeugen. Dieser Output des Algorithmus ist nicht notwendig determiniert, wird durch seine Regeln und deren Verkettungen jedoch stark vorstrukturiert. Zur besseren Lesbarkeit sprechen wir daher im Singular von dem AMS-Algorithmus.

Im Folgenden rekonstruieren wir die Entscheidungsregeln des AMS-Algorithmus aus dem veröffentlichten Material (Holl et al. 2018; Gamper et al. 2020). Unser Fokus liegt zunächst darauf, wie der AMS-Algorithmus potenzielle Empfänger ${ }^{2}$ von Leistungen vergleichbar macht, diese anschließend hinsichtlich ihrer Integrationschancen bewertet und sie schließlich in die Gruppe mit entsprechend niedrigen, mitt-

\footnotetext{
2 Gemeint sind Empfängerinnen und Empfänger. Dass im Weiteren das generische Maskulin verwendet wird, geht auf eine redaktionelle Entscheidung der KZfSS zurück. Verfasst wurde der Text in einer inklusiven Schreibweise.
} 
leren oder hohen Chancen einordnet. Im Anschluss an diese Rekonstruktion gehen wir auf analytische Merkmale des AMS-Algorithmus u. a. seinen Datenpragmatismus, seine sachliche und zeitliche Stabilitätsannahme und seine Fallorientierung, ein. Darauf aufbauend zeigen wir im dritten Abschnitt, wie das dergestalt operierende digitale Beobachtungsformat AMS-Algorithmus in die konkrete Entscheidungsarchitektur des AMS eingebunden ist und damit handlungsrelevant gemacht wird. Zuvor sind jedoch einige methodische Überlegungen notwendig.

Beim AMS-Algorithmus handelt es sich, wie bei vielen anderen Algorithmen, um einen proprietären Algorithmus, der für die soziologische Forschung nicht direkt zugänglich ist (vgl. dazu Bastin und Tubaro 2018). Das sich daraus ergebende Datenproblem wird jedoch zunächst dadurch entschärft, dass das Entwicklungsunternehmen des AMS-Algorithmus, die Synthesis Forschung GmbH, zwei Dokumentationen zur Verfügung stellt, die das Funktionieren des Algorithmus beschreiben (Holl et al. 2018; Gamper et al. 2020). Zudem wurde und wird die Einführung des Algorithmus durch eine Vielzahl an Stellungnahmen und Kontextualisierungen begleitet, die weitere Datenquellen darstellen. Obwohl der Algorithmus selbst nicht öffentlich ist und seine organisationspraktische Nutzung nicht beobachtet werden kann, erlauben diese Quellen es uns, nachzuvollziehen, wie der AMS-Algorithmus operiert und organisational in Entscheidungsarchitekturen eingebunden ist. Außer Frage steht, dass ethnografisch gewonnene Einsichten zukünftig wünschenswert sind, etwa um die Interaktionen und typischen Entscheidungssituationen zwischen Vermittler, Klient und Software zu analysieren. Für unser Forschungsinteresse sind die vorhandenen Dokumentationen jedoch adäquat, um am AMS-Algorithmus unser konzeptuelles Argument zum Verhältnis von Algorithmen und Organisationen zu entwickeln.

\subsection{Die Einführung des AMS-Algorithmus in den Österreichischen Arbeitsmarktservice}

Der Österreichische Arbeitsmarktservice ist ein Unternehmen des öffentlichen Rechts, dessen Ziele gemäß dem Arbeitsmarktservicegesetz durch das Bundesministerium für Arbeit, Familie und Jugend geregelt sind. Diese arbeitsmarktpolitischen Ziele kann der AMS relativ unabhängig von ministerialen Vorgaben umsetzen. Vor diesem Hintergrund beschlossen Verwaltungsrat und Vorstand des AMS im Frühjahr 2018, ein System zu entwickeln, um die Integrationschancen arbeitssuchender Personen bewerten zu können. Mit der Entwicklung wurde die bereits erwähnte und in Wien ansässige Synthesis Forschung $\mathrm{GmbH}$ beauftragt, nachdem diese sich zuvor in einer EU-weiten Ausschreibung durchsetzen konnte. Das Resultat dieser mehrmonatigen und insgesamt 1,8 Mio. Euro teuren Entwicklung war der AMSAlgorithmus oder das Arbeitsmarkt-Chancen-Modell, dessen erste Version die Synthesis Forschung im Oktober 2018 vorlegte (Holl et al. 2018). Eine aktualisierte Version folgte zu Beginn des Jahres 2020 unter der Bezeichnung Assistenzsystem AMAS (Gamper et al. 2020).

Mit Beginn des Jahres 2019 ging der AMS-Algorithmus in seine Erprobungsphase, die bis Ende 2019 dauerte. Vorgesehen war zudem eine Übergangsphase bis zum 1. Juli 2020, in der die Mitarbeiter des AMS darauf vorbereitet werden sollten, den 
Algorithmus zu nutzen. Aufgrund der Coronapandemie wurde die Einführung des AMS-Algorithmus in den Regelbetrieb jedoch verschoben. ${ }^{3}$

\subsection{Wie kategorisiert, vergleicht und bewertet der AMS-Algorithmus?}

Dieser Abschnitt wird aus einer konstruktivistischen Grundhaltung heraus rekonstruieren, wie der AMS-Algorithmus kategorisiert, vergleicht und bewertet. Wir gehen folglich davon aus, dass der AMS-Algorithmus zunächst eine Realität konstruiert, die es im Weiteren erlaubt, Erkenntnisse zu produzieren (Luhmann 1988, 2005). Die Veröffentlichungen der Synthesis Forschung sind hingegen von einer epistemologischen Grundhaltung getragen, die sich mit Desrosières (2001) als meteorologischer Realismus beschreiben lässt und die der Idee folgt, dass sich soziale Objekte wie physische Objekte messen lassen (vgl. auch Hacking 2001).

Komprimiert lässt sich die Operationsweise des AMS-Algorithmus wie folgt beschreiben. Zunächst kategorisiert der Algorithmus Arbeitssuchende und vergleicht diese anhand dieser Kategorien, zu denen Alter, Geschlecht, Staatsangehörigkeit und Arbeitsmarktbezirk gehören. Anschließend werden die Arbeitssuchenden im Hinblick auf ihre Integrationschancen bewertet, indem sie einer von drei sogenannten Chancengruppen zugeordnet werden. Im Unterschied zu Algorithmen, die auf maschinellem Lernen basieren, greift der AMS-Algorithmus auf „traditionelle“, sinnförmig verstehbare Kategorien zurück, um Personen vergleichbar zu machen. Anhand dieser Daten werden die Arbeitssuchenden mit einer sogenannten Basisgruppe verglichen, für die unterstellt wird, dass ihre Integrationschancen kaum eingeschränkt sind. Bei dieser Basisgruppe handelt es sich um „die Gruppe der jungen Männer mit höchstens [sic] Pflichtschulabschluss und österreichischer Staatsbürgerschaft. Sie haben keine Betreuungspflichten, sind nicht gesundheitlich beeinträchtigt und befinden sich in einem Arbeitsmarktbezirk 1, der zum RGS-Typ 1 gehört“ (Holl et al. 2018, S. 11). ${ }^{4}$ Diese Kategorisierung wird mittels logistischer Regression vorgenommen $^{5}$ und unterscheidet anhand der verschiedenen Merkmalsausprägungen 81.000 Konstellationen (Gamper et al. 2020, S. 13). Um die Integrationschancen einzelner Klienten zu bewerten, werden diese folglich als Merkmalssets dargestellt und mit allen anderen Klienten des AMS vergleichend in Beziehung gesetzt. Aus dieser Vergleichsbewegung resultiert ein konkreter Wert, der die Integrationschancen repräsentiert. Je nach Höhe des Wertes weist der Algorithmus die Arbeitssuchenden einer von drei Kategorien (Chancengruppen) zu: einer Gruppe mit niedrigen, einer mit mittleren und einer mit hohen Integrationschancen. Die Integrationswahrscheinlichkeit wird mittels unterschiedlicher Zeithorizonte operationalisiert. Wird für die

\footnotetext{
3 Zum Zeitpunkt des Verfassens dieses Beitrags (Winter 2020) ist umstrittener denn je, ob der AMS-Algorithmus tatsächlich wie vorgesehen eingeführt wird. Das konzeptionelle Argument des Aufsatzes berührt das konkrete Schicksal des AMS-Algorithmus jedoch nicht, da dieser vielmehr stellvertretend für vielfältige Versuche steht, öffentliche Organisationen mittels Algorithmen effizienter zu machen.

4 Arbeitsmarktbezirk 1 und RGS 1 bezeichnen Regionen, in denen das Modell von einer hohen Integrationswahrscheinlichkeit ausgeht.

5 Derartige logistische Regressionen sind ein zentraler Bestandteil von prädiktiven Technologien, die unter den Oberbegriffen der künstlichen Intelligenz und des maschinellen Lernens verhandelt werden (Boelaert und Ollion 2018).
} 
Niedrigchancen-Gruppe eine langfristige Integration angestrebt, ist für die Gruppe mit hohen Integrationschancen eine kurzfristige Integration maßgeblich. Die operative Logik des AMS-Algorithmus besteht also darin, Arbeitssuchende zunächst auf Basis digital verfügbarer Daten und selektierter Merkmale zu kategorisieren, anschließend auf dieser Basis zu vergleichen, um abschließend ihre Integrationschancen $\mathrm{zu}$ bewerten, wobei diese Chancen ihrerseits in ein ordinales Verhältnis gebracht werden (geringe, mittlere, hohe Chancen).

Mit der im Folgenden eingenommenen konstruktivistischen Perspektive erschließt sich die Kontingenz der Konstruktionsentscheidungen des Algorithmus. Um diese herauszuarbeiten, fokussieren wir auf vier ineinandergreifende Aspekte der Kategorisierung, des Vergleichs und der Bewertung des AMS-Algorithmus.

\subsubsection{Datenpragmatismus, Datenqualität und Kategorienkonstruktion}

Im Zuge der Digitalisierung exploriert der Österreichische Arbeitsmarktservice wie andere Organisationen auch, wie sich die eigenen Datenbestände unter den neuen technologischen Bedingungen nutzen lassen (Kallinikos et al. 2013; Kitchin 2014). Entsprechend prozessiert der AMS-Algorithmus eigene und verfügbare Daten, die zunächst zu anderen Zwecken gesammelt wurden. Ein zentrales Kriterium für die Wahl der verwendeten Daten ist damit weniger ihre Passung, sondern zunächst ihr schlichtes Vorhandensein. Basis der kategorialen Vergleichsbewegung des AMSAlgorithmus sind folglich bereits vorhandene Daten, die fallübergreifend erwartbar vorliegen (vgl. Graeff und Baur 2020) und von hinreichender Qualität für statistische Auswertungszwecke sind. Dieser Umgang mit Daten lässt sich im Anschluss an Desrosières (2001) und Spiegelhalter (2019) als organisationaler Datenpragmatismus bezeichnen, der sich fundamental in die soziale Vergleichsbewegung einschreibt, mittels derer Arbeitssuchende zueinander in Beziehung gesetzt werden. Mit Pragmatismus ist nicht gemeint, dass Daten und deren Kategorienschemata leichtfertig modelliert würden, sondern dass das Vorliegen und die Verfügbarkeit organisationaler Daten es legitimieren, diese Daten bei aller Problematik (,,bad data“) zu nutzen (McCallum 2013). Dies zeigt sich im Fall des AMS sowohl in der Zusammenstellung der Datenkategorien als auch im Grad der Differenzierung, mit dem Ausprägungen von Merkmalen in den Algorithmus eingehen. So verarbeitet der AMS-Algorithmus vorwiegend nominalskalierte Daten. Diese nichtmetrischen und wenig differenzierten Daten lassen jedoch nur begrenzte Auswertungsmöglichkeiten zu. Die Logik des Zugriffs auf vorhandene Daten zeigt sich zudem deutlich an den drei Datenkategorien, auf die der AMS-Algorithmus seine Berechnungen stützt. Bei der ersten Datenkategorie handelt es sich um persönliche Merkmale, wie Geschlecht, Alter, Staatsbürgerschaft, Ausbildung, Betreuungspflichten und gesundheitliche Einschränkungen. Die zweite Datenkategorie stellen Daten zur aktuellen Dauer der Arbeitslosigkeit dar. Die dritte Datenkategorie ist die berufliche Historie der Klienten, die mittels des bisherigen Berufs, des Umfangs der beruflichen Tätigkeit, der regionalen Verankerung und der bisherigen Arbeitslosigkeiten ermittelt wird. Vergegenwärtigt man sich darüber hinaus das Ziel, Integrationswahrscheinlichkeiten konkreter Klienten zu bewerten, also einzelfallspezifische Prognosen zu erstellen, ist es überraschend, dass der aktuelle Beruf in dieser dritten Datenka- 
tegorie lediglich mittels der Ausprägungen Dienstleistung und Produktion erfasst wird. Auch gehen gesundheitliche Beeinträchtigung nur in zwei Ausprägungen in die Berechnung ein. Gerade weil die Potenziale von Big Data in der optimierten Nutzung menschlich nicht prozessierbarer Informationsmengen gesehen werden, ist diese reduzierte Modellierungsweise von Beruf und Gesundheit für die Frage, wie die Integrationschancen von Klienten bewertet werden, bemerkenswert.

Die Datenkategorien lassen erkennen, dass der AMS-Algorithmus mit dem Problem unvollständiger Daten umgehen muss, da für Personengruppen wie Jugendliche, Migranten oder Personen mit fragmentierten Erwerbsbiografien oft nur partielle Daten vorliegen. Diesem Problem begegnet die Modellierung zum einen durch die Nominalskalierung der verfügbaren Variablen und zum anderen, indem sie zwei Teilpopulationen bildet: eine, für die vollständige Daten vorliegen (voll valide Population), und eine, für die nur unvollständige Daten vorliegen (teilvalide Population).

Vergleicht man den AMS-Algorithmus mit nichtalgorithmischen Bewertungen, zeigt sich, dass der Algorithmus mittels der beschriebenen Datenkategorien eine eng umrissene und aus vorhandenen organisationalen Daten komponierte Realität konstruiert, um Integrationschancen zu beurteilen. Diese Realität ist ein abgedichteter Vergleichs- und Kriterienraum. Vergleicht man diesen Vergleichs- und Kriterienraum mit dem Ermessen von Vermittlern, die ohne algorithmische Entscheidungshilfe Integrationschancen bewerten, ist zunächst erkennbar, dass auch deren Ermessen vorstrukturiert ist - etwa durch formale Vorgaben, professionelle Normen oder Vorstellungen von guten, schlechten und schwierigen Klienten (Weinbach 2014). Im Unterschied zum algorithmischen Bewertungs- und Vergleichsraum ist dieser jedoch kategorial weder einheitlich noch abgedichtet; er bleibt bei aller Vorstrukturierung bedingt offen dafür, irritiert und beeinflusst zu werden und ist damit ebenfalls bedingt offen für zusätzliche Kategorien oder die Notwendigkeit, bestehende Kategorien zu relativieren. Diese bedingte Responsivität ist nicht per se voroder nachteilig für Klienten, sondern zunächst lediglich eine strukturelle Differenz im Umgang mit Kategorien in der vergleichenden Bewertung.

\subsubsection{Bewertungskontinuität}

Der AMS-Algorithmus dichtet seinen Vergleichsraum nicht nur sachlich, sondern auch zeitlich ab. Dies zeigt sich darin, dass der AMS-Algorithmus als prädiktiver Algorithmus die Vergangenheit in die Zukunft extrapoliert (vgl. dazu grundsätzlich Hagendorff und Wezel 2019). Diese Extrapoliation zeigt sich in der Prämisse, dass vergangene Beeinträchtigungen der Integrationschancen fortdauern werden und deshalb bei der Bewertung der gegenwärtigen Integrationschancen zu berücksichtigen sind (vgl. Gamper et al. 2020, S. 71). Wie Braudel (1958, S. 746) mit Blick auf mathematische Modelle schon früh bemerkt hat, wird auf diese Weise Vergangenheit als Zukunft fortgeschrieben, da die Vergangenheit als „très longue durée“ unterstellt wird. Problematisch kann diese Fortschreibung wiederum nicht nur in Hinblick auf einzelfallspezifische Entwicklungsdynamiken, sondern auch in dynamischen Umwelten werden. Während wiederkehrende Dynamiken, wie saisonale Schwankungen, in algorithmischen Vergleichsdimensionen berücksichtigt werden können, ist dies bei punktuellen Disruptionen (z. B. veränderten Einwanderungsbestimmungen, 
Fluchtbewegungen, Pandemien) oder dem langsamen Wandel von Berufszweigen nicht möglich.

\subsubsection{Vergleichsrichtung Fallorientierung}

Digitale Beobachtungsformate versprechen eine hohe Personalisierung der Fallbearbeitung. Exemplarisch hierfür steht der Bereich der personalisierten Medizin, in dem Behandlungen zunehmend an individuellen Merkmalen orientiert vorgenommen werden können. Die Erwartung, die Fallbearbeitung personalisieren zu können, zeigt sich aber auch am AMS, wie ein Interview mit dem AMS-Vorstand, Johannes Kopf (2018), in der Zeitung Der Standard illustriert:

„Dieses IT-Tool prognostiziert mithilfe hochkomplexer mathematischer Modelle monatlich neu die rechnerisch aktuellen Arbeitsmarktchancen jedes einzelnen arbeitssuchenden Menschen. Damit werden aus dessen bisherigem Erwerbsverlauf sowie aus einer Vielzahl anderer persönlicher Kriterien wie etwa Alter, Ausbildung, Region, Geschlecht oder Betreuungspflichten die jeweils individuellen Chancen auf einen baldigen Arbeitsbeginn mit erstaunlich hoher Trefferquote vorhergesagt."

Für algorithmische Entscheidungshilfen in personenbezogenen Organisationen wie dem AMS ergibt sich damit auch für algorithmische Beobachtungsformate ein bekanntes Spannungsfeld: das zwischen Fall- und Biografieorientierung. Mit Carol Heimer (2001) lassen sich beide Orientierungen als kulturell-kognitive Perspektiven begreifen, die die Identität von Handlungen, Akteuren und Objekten strukturieren (Heimer 2001, S. 48). Während die biografische Perspektive narrativ die kausale Einheit von Dingen betont (die Provenienz eines Kunstwerks, das Erleben von Krankheit durch Laien), wird diese Identität in Fallorientierungen durch den Vergleich von Dingen mit anderen Dingen gewonnen (das Highlight in einer Ausstellung, die Diagnosen von Ärzten). In weniger durchformalisierten Situationen, wie beispielsweise im Bereich der Erziehung, dominieren oft biografische Orientierungen, während in formal routinisierten Situationen, wie Gerichtsverfahren (Schwarting 2020), Fallorientierungen stärker anzutreffen sind. Empirisch kommt es regelmäßig auch zu Mischformen, sei es im Jugendstrafrecht, das individuelle Entwicklungsperspektiven berücksichtigt oder in Fallbearbeitungen über längere Zeiträume hinweg, etwa wenn eine Arbeitssuchende zunächst als Fall, später dann biografieorientierter vermittelt wird (als Gewährung einer zweiten Chance, einer Bewährungsprobe, der Erfüllung eines lang gehegten Qualifizierungswunsches).

Prädiktive algorithmische Systeme legen ebenso wie andere statistische Verfahren (Wobbe 2012) Fall- statt Biografieorientierung nahe, da sie Individualität als spezifische Ausprägung eines Merkmalssets entwerfen. Eine vorrangige Fallorientierung lässt sich auch beim AMS-Algorithmus beobachten. Diese resultiert aus der beschriebenen Vergleichsbewegung, die Klienten als Merkmalssets begreift, vergleicht und bewertet. Der AMS-Algorithmus entwirft seine Fälle damit vorrangig als stabil, da, mit Ausnahme weniger Kategorien wie der der Vorkarriere, kaum Kategorien genutzt werden, deren Ausprägungen individuell änderbar sind. Diese Tendenz, arbeitssuchende Personen als Fälle zu behandeln, wird dadurch begünstigt, dass der 
AMS-Algorithmus, wie beschrieben, auf der Basis von organisationsseitig vorhandenen Daten operiert, die ihrerseits fallorientiert erzeugt wurden (organisationaler Datenpragmatismus). Biografisches Wissen scheint demgegenüber nicht systematisch berücksichtigt zu werden. Anders als Unternehmen wie Amazon, die durch selbst gestaltete digitale Kontexte automatisierbar detaillierte Nutzerinnendaten generieren, dokumentieren personenbezogene Organisationen (Klatetzki 2010) wie der AMS ihre Fälle mittels vergleichsweise gering und grob aufgelöster Daten. Zugleich lassen sich biografische und narrativ verfasste Daten, die z. B. in Freitextfeldern anfallen, vergleichsweise schwer in algorithmische Systeme der Entscheidungsunterstützung integrieren.

Die Bewertungs- und Vergleichsrichtung des AMS-Algorithmus ist damit stark fallorientiert und zugleich individualisierend. Individualisierung als Richtung der Bewertung und des Vergleichs meint hier nicht, dass individuelle Merkmale in besonders starkem Maße berücksichtigt werden. Von einer individualisierenden Richtung sprechen wir, weil hier durch das algorithmische Modell strukturelle Diskriminierungen, etwa durch die Übernahme von Betreuungspflichten, in individuelle Integrationschancen übersetzt werden (vgl. dazu auch Barnard und Fourcade 2021, in dieser Ausgabe). Solche Prozesse der Individualisierung sozialer Probleme sind gleichwohl seit Langem aus Sozialverwaltungen auch ohne algorithmische Entscheidungsunterstützung bekannt (Wolff und Bonß 1979). Gleichzeitig ist diese Individualisierung durch algorithmische Entscheidungsunterstützung nicht zwangsläufig, sondern Ausdruck organisationaler Entscheidungen darüber, wie die vorliegenden Daten algorithmisch prozessiert werden sollen. So ließe es die Datenbasis des AMS auch zu, die Daten arbeitssuchender Personen für eine Rekonstruktion struktureller Benachteiligungsmuster auf dem regionalen Arbeitsmarkt und in der Fallbearbeitung durch den AMS zu nutzen. Derartige explorative Nutzungen des Datenmaterials könnten dann etwa Hinweise auf blinde Flecken der Förderung, z. B. von multipel diskriminierten Klienten geben und die Entwicklung von Gegenmaßnahmen erleichtern. Eine diesbezügliche Frage könnte beispielsweise lauten, in welchen Regionen sich Betreuungspflichten besonders negativ auswirken. Die Kontextualisierung des Ergebnisses (Suche nach Erklärungsfaktoren) kann dann situiert und unter Nutzung digitaler und nichtdigitaler, verfügbarer und neu zu schaffender Datenkorpora erfolgen. Würde man die Daten entsprechend nutzen, um das Verhältnis von Organisation und Algorithmus neu zu konfigurieren, würde der Algorithmus Vergleichsräume öffnen, statt diese zu schließen. Damit ließe sich auf struktureller Ebene mutmaßlich die Integrationswahrscheinlichkeit von Klientengruppen langfristig erhöhen.

Zusammenfassend formuliert prozessiert der AMS-Algorithmus zwar strukturelle Diskriminierungsmuster, produziert aber einen Output, der die Effekte dieser Diskriminierung individualisiert, indem er einen für einzelne Klienten gültigen Output produziert, mit dem Unterstützungsmaßnahmen eröffnet oder verschlossen werden.

\subsubsection{Naturalisierte Kategorien}

Der AMS-Algorithmus nimmt mit der ordinalskalierten Unterteilung von niedrigen, mittleren und hohen Integrationschancen eine kommunikativ sehr anschlussfähige, da intuitiv plausible und fast natürlich anmutende Kategorisierung vor. Entsprechend 
wenig begründungsbedürftig erscheint diese Trias im Kommentar des Vorstandes des AMS:

„Die Einführung des neuen AMS-Algorithmus entspricht unserem Wunsch, die Effizienz jeder einzelnen Beratung, aber auch die Effektivität des gesamten AMS zu steigern. Sinnvollerweise werden dafür unsere Kundinnen und Kunden künftig in drei Gruppen erfasst" (Kopf 2018).

Ebenso wie bei Vier-Felder-Matrizen (Pollock und D'Adderio 2012) handelt es sich auch bei der Trias von niedrig, mittel und hoch um ein institutionalisiertes Schema der Klassifikation von Sachverhalten und Personen. Analytisch sind die kontingenten Entscheidungen, die dieser Dreierunterscheidung zu Grunde liegen jedoch bemerkenswert. Diese Kontingenz zeigt sich, wie unter 2.2 erläutert, daran, dass sich das Erfolgsmaß für gelungene Integration zwischen den Gruppen unterscheidet, dafür die beiden Randgruppen (niedrige und hohe Chancengruppe) unterschiedliche Zeithorizonte verwendet werden. Zudem wird die Niedrigchancen-Gruppe mit einem 25-prozentigen Integrationschancenwert definiert, wohingegen dieser Wert bei der Hochchancen-Gruppe $66 \%$ beträgt. Hier wird deutlich, dass ,big categories“ wie die Dreierunterscheidung erst durch ,little analytics“ (Amoore und Piotukh 2015), also Grenz-, Schwellen- und Restkategorisierungen bei algorithmischen Systemen, ins Werk gesetzt werden. Diese ,little analytics“, das Zuschneiden der Zeithorizonte und die Definition der Schwellenwerte, sind nicht einfach Notwendigkeiten der Modellierung, sondern besagte kontingente Entscheidungen, die als ,instruments of perception“ die Realitätskonstruktion des Algorithmus prägen: „[T]hey carve out images; reduce heterogeneous objects to a homogeneous space; and stitch together qualitatively different things such that attributes can be rendered quantifiable“ (Amoore und Piotukh 2015, S. 348). Die Klarheit der Dreierunterscheidung der Integrationschancen wird durch das Operieren der ,little analytics“ ermöglicht. Weil deren Operieren jedoch nicht in der Benennung sichtbar gemacht wird, verfestigt sich der Eindruck einer plausiblen symmetrischen Kategorisierung von Arbeitssuchenden. Die Veränderbarkeit dieser ,little analytics“, etwa die Verringerung der Dauer, mit der Klienten beschäftigt sein müssen, um als integriert zu gelten, eröffnet zugleich für die Organisation die Möglichkeit, ihre Klientenumwelt qua algorithmischer Kategorisierung bearbeitbar zuzuschneiden, denn veränderte Schwellenwerte vergrößern und verkleinern die Chancengruppen. Dekonstruiert man die Kategorien weiter, zeigt sich, dass gerade die Bestimmung der Hauptzielgruppe, der mittleren Chancengruppe, am schwersten greifbar ist. Diese mittlere Chancengruppe wird in den Berechnungen des Modells nicht positiv bestimmt, sondern ausschließlich ex negativo kreiert; sie ist der ,errechnete Rest“ zwischen der Hoch- und der Niedrigchancen-Gruppe (Gamper et al. 2020, S. 59). Zugleich ist dieser Rest interpretativ schwer fassbar, da für die Hoch- und die Niedrigchancen-Gruppe Integrationserfolg uneinheitlich definiert ist (kurz- und langfristige Perspektive).

Der Eindruck plausibler und scheinbar selbstverständlicher Kategorien wird nicht nur durch institutionalisierte Dreierunterscheidungen, sondern auch über naturalisierende Semantiken erzeugt. In diesem Fall betrifft dies die Rede von Meilensteinen der Fallbearbeitung. Meilensteine bezeichnen die einzelnen Zeitpunkte, zu denen Klientinnen algorithmisch bewertet werden (Holl et al. 2018, S. 3). Die erste Be- 
wertung wird zu Beginn des Geschäftsfalles vorgenommen; quartalsweise wird dann bis zum 24. Monat erneut kategorisiert und anschließend nochmals nach 30, 36 und 48 Monaten. Mittels dieser aus dem Projektmanagement bekannten Semantik werden kontingent gesetzte Zeitpunkte der algorithmischen (Neu-)Bewertung in die Logik der Fallbearbeitung integriert. Dabei evoziert die Begrifflichkeit des Meilensteins die Vorstellung, Fälle würden aus sich heraus stabil und linear fortschreiten. Anders als bei Wanderungen und im Projektmanagement repräsentieren Meilensteine hier jedoch keinen Fortschritt, etwa weil ein bestimmter Ort oder ein kritischer Projektschritt erreicht wird, sondern lediglich kontingent gesetzte Zeitpunkte, zu denen Klienten erneut kategorisiert werden.

\subsubsection{Intervention und Koproduktion als fehlende Kategorien - organisationaler Selbstbezug im Algorithmus}

Wie dargelegt, beeinflussen die Leistungsmöglichkeiten und Versprechen innovativer digitaler Technologien, wie Organisationen Daten wahrnehmen und nutzen. Wir haben diesbezüglich gezeigt, dass Organisationen dazu tendieren, qualitativ problematische, aber vorhandene Datenbestände pragmatisch zu nutzen, anstatt zu versuchen, diese neu zu erzeugen oder von einer algorithmischen Nutzung abzusehen, wenn die Passung der Daten zu dem zu bearbeitenden Problem begrenzt ist. Als eine Quelle unvollständiger Daten haben wir zudem externe Faktoren benannt, z. B. fragmentierte Erwerbsverläufe, die lückenhafte Datensätze wahrscheinlich machen. Gleichzeitig beeinflussen aber auch organisationsinterne Faktoren die Datenqualität, etwa weil bestimmte Daten in der Organisation nicht verlässlich dokumentiert werden. Dies verdeutlicht, dass sich Organisationen früh und uneinheitlich nicht nur in algorithmische Modelle, sondern auch in die von diesen genutzten Datenkorpora einschreiben. Diese Einschreibungen geschehen nicht nur „positiv“, indem Daten selektiert werden, sondern auch „negativ“, indem Organisationen Leerstellen und Aussparungen produzieren (Frickel 2014).

Solche Aussparungen verdienen analytische Aufmerksamkeit, da sie dafür sensibilisieren, dass sich in Algorithmen auch organisationale Selbstbezüge einschreiben. Im Fall des AMS-Algorithmus ist eine Aussparung besonders bemerkenswert: Der Algorithmus blendet den intervenierenden Charakter einer Arbeitsvermittlung bei der Zuweisung von Integrationschancen weitgehend aus, behandelt also die Integrationschance als weitgehend unabhängig von den Leistungen der Arbeitsvermittlung. Vorangegangene Unterstützungsleistungen des AMS scheinen lediglich an wenigen Stellen auf, etwa dort, wo zurückliegende Leistungsbezüge der arbeitssuchenden Person über die „Vorkarriere“ in die Berechnung integriert werden. In der Sprache des meteorologischen Realismus gesprochen, beobachtet der AMS nicht nur Arbeitsmärkte, sondern interveniert in das vorherzusagende Phänomen, ist also reaktiv qua Einbindung in Entscheidungsstrukturen. Die Modellierung der Integrationswahrscheinlichkeit operiert zwar mit einer Datenbasis, die auf Klientendaten des AMS basiert, aber allein deswegen spiegelt sich in diesen Daten nicht automatisch der Interventionscharakter des AMS, also seine potenziell aktive Rolle bei der Reintegration in den Arbeitsmarkt wider. Im Gegenteil: Der organisationale Selbstbezug, der sich durch die Selektion oder den Ausschluss von Datenkategorien hier in den 
Algorithmus einschreibt, schaltet zentrale Interventionen des AMS in das Problem Arbeitsmarktintegration stumm.

Bei diesen stummgeschalteten Interventionen handelt es sich in zentraler Hinsicht um die Bedeutung der Vermittler und der Klienten dafür, dass Beratung gelingt. Selbst wenn die Möglichkeiten der situativen Aushandlung der Vermittler algorithmisch vorstrukturiert und damit reduziert werden, verschwinden Aushandlungsräume nicht. Entsprechend ist auch im Fall des AMS davon auszugehen, dass der Algorithmus nicht spezifische Maßnahmen zuweist, sondern qua Kategorisierung Korridore von Maßnahmen geöffnet oder verschlossen werden. So bleiben typische Formen der Zusammenarbeit zwischen Mitarbeiter und Klient, also Grenzstellenarrangements (Büchner 2018b, S. 152 ff.) auch bei algorithmischer Entscheidungsunterstützung weiter relevant.

Intervention wird auch in einer zweiten Hinsicht im AMS-Algorithmus ausgeblendet: Der Algorithmus invisibilisiert nicht nur die Rolle der Vermittler, sondern auch sich selbst als Zuweisungsinstanz. Für all diese Umstände lassen sich technische, datenschutzrechtliche und modellierungspraktische Erklärungen finden. Dennoch bleibt die dargelegte Beobachtung relevant, dass sich in prädiktive Algorithmen ein organisationaler Selbstbezug qua selektierter, aber auch qua ausgeschlossener und fehlender Daten einschreibt. Dieser Selbstbezug kann, wie in diesem Falle, darin bestehen, dass Interventionsanteile am vorherzusagenden Phänomen (Integrationswahrscheinlichkeit) nicht ein-, sondern weitgehend ausgeblendet werden. Gerade wenn Algorithmen mit dem Ziel der Effektivitäts- und Effizienzsteigerung in intervenierend und koproduktiv operierenden personenbezogenen Organisationen eingesetzt werden, muss ein weitgehendes Ausblenden eigener Leistungsanteile im organisationalen Selbstbezug des Algorithmus kritisch betrachtet werden. Dies impliziert keine grundsätzliche Ablehnung oder Befürwortung von Algorithmen, sondern den Hinweis, dass analysiert werden sollte, inwiefern Algorithmen Vorstellungen der Abhängigkeit oder Unabhängigkeit ihrer Objekte etablieren und stabilisieren, hier hinsichtlich der Beziehung zwischen Klient und Vermittler sowie den getroffenen Maßnahmen und der Integration in den Arbeitsmarkt. Derartige Autonomieannahmen sind je nach Organisationstyp, dem zu lösenden Organisationsproblem und dem formalisierten Problem, das der Algorithmus zu lösen beabsichtigt, unterschiedlich plausibel. Die Erkennung von Tumormustern durch Algorithmen unterscheidet sich von der Erkennung der Integrationswahrscheinlichkeit von Arbeitssuchenden. Für soziologische Beobachterinnen verschwindet dieser Unterschied nicht, nur weil er algorithmisch kaum oder nicht mehr auftaucht.

\section{Wie der AMS-Algorithmus actionable wird}

Im vorherigen Abschnitt haben wir rekonstruiert, wie das algorithmische Modell kategorisiert, bewertet und vergleicht. In diesem Abschnitt zeigen wir, wie der AMSAlgorithmus in die Entscheidungsprozesse des AMS eingebunden ist und dass es diese konkrete Einbindung ist, die den AMS-Algorithmus actionable macht. Dies geschieht, indem der Output des Algorithmus, also die Bewertung arbeitssuchender Personen durch deren Zuordnung zu einer von drei Kategorien oder Chancengruppen, in der 
organisationalen Entscheidungsarchitektur als Anschlussstelle und Auslöser für Folgeprogramme verankert wird. Damit sensibilisieren wir dafür, dass es nicht allein und nicht primär die algorithmisch bearbeitete Klassifikationssituation per se ist, die einen sozialen Unterschied macht, sondern die Relation zu vor- oder nachgelagerten Entscheidungsprozessen. Entsprechend werden wir im Folgenden den AMSAlgorithmus in Relation zu den Elementen der organisationalen Programmstruktur charakterisieren.

\subsection{Zur Einbindung algorithmischer Modelle in Organisationen}

Algorithmische Modelle erlangen auf unterschiedliche Art soziale Relevanz. Während diese bei Dating-Apps (Illouz 2015, S. 319 ff.; Peetz 2021, in dieser Ausgabe) oder Streamingdiensten über ihre gesellschaftliche Verbreitung und breite individuelle Nutzung entsteht, ist dies bei algorithmischer Entscheidungsunterstützung zur Distribution öffentlicher Güter und Ressourcen anders. Sowohl Business Analytics als auch Kategorisierungssysteme wie der AMS-Algorithmus werden nicht nur in Organisationen genutzt, sondern gewinnen erst durch Organisationen Relevanz. Erst indem ihre Vergleiche, Bewertungen und Kategorisierungen als Praktiken in organisationale Entscheidungsarchitekturen eingelassen werden, gewinnen diese relative Stabilität und damit die Möglichkeit, Unterschiede für andere, relativ stabil erzeugte Entscheidungen zu machen. Mit dieser soziologischen Fragerichtung verschieben wir die aktuell leitende analytische Differenz von der Unterscheidung Individuum/ Algorithmus oder menschliches Entscheiden/algorithmisches Entscheiden hin zur Unterscheidung Organisation/Algorithmus. Diese Perspektive trägt dazu bei, hybride Entscheidungszusammenhänge analytisch tiefenschärfer ausleuchten zu können, indem die Organisiertheit von Entscheidungssituationen gegenüber der Annahme in den Fokus rückt, der Umgang mit Algorithmen sei primär von Personen oder der Mensch-Algorithmus-Anordnung in idealtypischen Situationen abhängig (vgl. Danaher 2016; Petersen et al. 2020; Kleinberg et al. 2018; O’Neil 2017).

Die beschriebene Fragerichtung nach dem Wie der organisationalen Einbettung des Algorithmus basiert darauf, dass Organisationen digitale Beobachtungsformate wie den AMS-Algorithmus kontingent und damit heterogen einbetten. Im Fall des AMS-Algorithmus wird eine punktuelle algorithmische Ergänzung der Entscheidungsarchitektur, gewissermaßen eine algorithmische Injektion, vorgenommen. Hierbei wird der Algorithmus konkret in bereits bestehende Entscheidungswege der Fallbearbeitung eingepasst. Demgegenüber erfordert die Einführung umfassender Systeme wie SAP und Prepolicing-Plattformen, dass Organisationspraktiken und Entscheidungswege neu konfiguriert werden, wodurch sie einen weitergehenden organisationalen Umbau einleiten (Pollock und Williams 2008; Conrad 2017).

\subsection{Stabile Kopplungen als kontingente Einbettung}

Die Einbindung des AMS-Algorithmus in die Entscheidungsarchitektur des AMS gestaltet sich erstens nicht lose und flexibel, sondern stabil in Bezug auf Folgeoperationen. Zweitens begünstigt die organisationale Situierung der Entscheidungssituation deren stabile Anbindung an Folgeentscheidungen. 
Der stabile Bezug auf Folgeoperationen zeigt sich im AMS-Fall darin, dass die algorithmische Kategorisierung Unterstützungsmöglichkeiten eröffnet und verschließt und damit das konkrete Problem des „Wie weiter?“ in der Fallbearbeitung löst. Insofern nutzt der Algorithmus ,,past data to answer immediate practical questions " und ist nicht Teil der „scientific systems that seek to understand how the world works“" (Spiegelhalter 2019, S. 145; Herv. StB und HD). Damit ist der AMS-Algorithmus ein Instrument, um Folgeentscheidungen der Fallbearbeitung zu strukturieren oder vorzustrukturieren. Die praktische Frage auf der Ebene des Einzelfalls lautet: Welches Unterstützungsspektrum wird für Klienten eröffnet oder verschlossen? Die praktische Frage auf Organisationsebene lautet: Wie werden begrenzte organisationale Leistungskapazitäten distribuiert?

Die Kontingenz dieser stabilen Einbettung des Algorithmus zeigt sich im Kontrast zu einer flexiblen Einbettung von Algorithmen. Ein Beispiel hierfür sind Business Analytics, also Systeme zur Exploration von Datenkorpora (Barbour et al. 2018). In diesem Fall wird die Handlungsrelevanz von Algorithmen nicht durch feste Kopplungen an Anschlussprozesse generiert, sondern dadurch, dass sie unterschiedliche Anschlussentscheidungen offenhalten und ermöglichen. Algorithmische Resultate werden so zu Inspirationsgebern, um neue Maßnahmen zu entwickeln, zum Anlass, Daten vertieft zu analysieren oder Organisationsdaten mit weiteren Datenquellen zu verknüpfen. Wie andere quantifizierte Neuigkeiten können sie ebenso beim nächsten Meeting lediglich ritualisiert zur Kenntnis genommen werden. Ihre flexible Kopplung erlaubt es, Überschussmöglichkeiten zu generieren, weil an den algorithmischen Output unterschiedlich angeschlossen werden kann.

Während Organisationen algorithmische Systeme flexibel oder fest in Entscheidungsarchitekturen einbinden können, zeigt das Beispiel des AMS-Algorithmus, dass Algorithmen aus organisationalen Entscheidungsarchitekturen auch herausgelöst und rekontextualisiert werden können. So veröffentlichte die österreichische Zeitung Der Standard im Oktober 2018 eine Simulation des AMS-Algorithmus mit eigenem Interface (Kienzl und Szigetvari 2018). Interessierte können mit dieser ihre kurzfristige Integrationswahrscheinlichkeit berechnen lassen. Während solche Gamifizierungen oft als die Einführung von spielerischen Elementen in nichtspielerische Handlungskontexte verstanden werden (Walz und Deterding 2015), wird der AMS-Algorithmus hier gewissermaßen durch inverse Gamifizierung spielerisch erlebbar: Weil der Algorithmus aus seiner Einbindung in die Entscheidungsarchitektur des AMS herausgelöst und damit von seiner sozialen Relevanz im Ursprungskontext entlastet wird, können sich Personen folgenlos algorithmisch bewerten lassen. Die AMS-Kategorisierungen werden dadurch freigestellt von Entscheidungsrelevanz; die Berechnung der Integrationswahrscheinlichkeit lässt sich beliebig häufig zu selbstgewählten Zeitpunkten wiederholen. ${ }^{6}$

Nachdem wir die Kontingenz der stabilen Einbettung von Algorithmen in organisierte Entscheidungsarchitekturen aufgezeigt haben, gilt es auf einen zweiten Aspekt hinzuweisen, der die enge Kopplung des AMS-Algorithmus an Folgeentscheidungen über Unterstützungsleistungen begünstigt: Die organisationstypische Situierung der Entscheidungssituation. Während Fourcade und Healy (2013) mit ihrem Begriff

\footnotetext{
${ }^{6}$ Für die aufschlussreiche Diskussion zur Gamifikation danken wir Matthias Bottel.
} 
der classification situations darauf hinweisen, dass privatwirtschaftlich entwickelte Klassifikationsinstrumente marktförmige Situationen kreieren, geht es bei der uns hier interessierenden Form der Situierung um eine organisationale Situierung, also um eine, die auf Regelmäßigkeiten von Entscheidungssituationen in Organisationen abstellt. Solche Regelmäßigkeiten prägen strukturell die Entscheidungssituationen von Nutzern von Algorithmen, hier der Vermittler in Arbeitsvermittlungen. Diese Regelmäßigkeiten entstehen etwa durch zu bearbeitende Fallzahlen, durchschnittliche Bearbeitungszeiten, durch Eingabezwänge und -möglichkeiten des Interface sowie durch die typische soziale Form, mit der wiederholt entschieden wird (z.B. allein oder zu zweit, von einer festen Vermittlerin oder mit wechselnden Zuständigkeiten).

Diese Fragen der organisationalen Situierung lassen sich im Fall empirischer Feldzugänge präzise beantworten. Da diese im untersuchten Fall nicht zugänglich sind, lassen sich nur näherungsweise Vermutungen anstellen: Anzunehmen ist, dass die organisationale Situiertheit im Fall des AMS stabile Kopplungen des Outputs mit Entscheidungssituationen begünstigt und damit, dass die Vermittler strukturell kaum in der Lage sind, die algorithmische Bewertung arbeitssuchender Personen zu prüfen. Während dies die Möglichkeiten der Vermittler, individuelle Lösungen im Einzelfall auszuhandeln, nicht beschränkt (siehe oben), senkt die Zuständigkeit einer Fachkraft für mehr als 250 Arbeitssuchende ${ }^{7}$ die Wahrscheinlichkeit, den algorithmischen Output kontinuierlich zu prüfen. Ebenso macht dies eine lose oder uneinheitliche Kopplung des algorithmischen Outputs wenig wahrscheinlich. Anders gewendet: Damit unter diesen Bedingungen organisationaler Situierung eine lose Kopplung, etwa in Form der Prüfung jeder algorithmischen Kategorisierung, wahrscheinlich wird, müssten sehr spezifische und umfassende Vorkehrungen getroffen werden, insbesondere, müsste neben der Datenkompetenz der Fachkräfte vor allem zusätzliche Zeit eingeräumt werden, um algorithmische Bewertungen überhaupt prüfen zu können. Solche Ermöglichungsbedingungen stünden jedoch im Widerspruch zu Effizienzversprechen, die die Einführung algorithmischer Entscheidungsunterstützung begleiten.

\subsubsection{Priorisierung durch frühe Einbindung in Entscheidungsarchitekturen und standardisierte einseitige Rekategorisierung}

Das Problem, über die Verteilung begrenzter Ressourcen entscheiden zu müssen, wird durch Organisationen auf unterschiedliche Weisen gelöst. So haushalten Grenzstellen (Luhmann 1999; Büchner 2018b) und Street-Level-Bürokraten (Lipsky 1980; Petersen et al. 2020) in der Bearbeitung von Fällen mit ihrer Initiative, dosieren also ihr Eingreifen, und wirken so als vermittelnde Instanzen zwischen Organisation und Umwelt. Ein weiteres Organisationsprinzip sind Priorisierungen, also Dringlichkeitsbewertungen von internen und externen Ereignissen. Von der Entscheidungsarchitektur her betrachtet, werden dabei Programmelemente in Entscheidungsketten nach vorn verlagert und mit Relevanz für Folgeentscheidungen ausgestattet, indem sie etwa als Auslöser für Folgeprogramme fungieren. Diese frühe Einbettung in Ent-

\footnotetext{
7 Damit gemeint sind Fälle, in denen Arbeitssuchende länger als 3 Monate arbeitslos sind (Fanta 2018).
} 
scheidungsketten wird auch beim AMS-Algorithmus eingesetzt: Die Kategorisierungen, die der Algorithmus vornimmt, werden direkt zu Beginn der Fallbearbeitung relevant gemacht und fungieren in der Folge als Masterkategorie, die Zugänge zu Ressourcen eröffnet oder verschließt.

Die Kontingenz der frühen und prominenten Einbettung des AMS-Algorithmus ist als solche erkennbar, wenn man sich alternative Formen der organisationalen Einbettung vergegenwärtigt. So ließe sich die Relevanz des Merkmals Integrationswahrscheinlichkeit ebenso an nachgeordneter Position algorithmisch integrieren, etwa als optionales Entscheidungskriterium, das Vermittler konsultieren können, um darüber zu entscheiden, wer an besonders knappen Integrationsmaßnahmen teilnehmen sollte. Der AMS-Algorithmus hingegen kreiert durch seine frühe organisationale Einbindung die drei Gruppen als Masterkategorien. Die hohe soziale Relevanz dieser algorithmischen Kategorisierung wird weiter dadurch befördert, dass die (Re-)Kategorisierung standardisiert zu fixen Zeitpunkten stattfindet (an den Meilensteinen). Alternativ könnten algorithmische (Re-)Kategorisierungen auch so zugeschnitten werden, dass sie zeitlich ungebunden durch Vermittler und Klienten initiiert werden können. Insofern distribuiert nicht der Algorithmus, sondern die Organisation Kategorisierungsanlässe, agiert also sowohl als Instanz zur Stiftung von algorithmischen Bewertungsanlässen als auch als Instanz ihrer Verknappung. Die vieldiskutierten Möglichkeiten der Überschreibung algorithmischer durch menschliche Bewertungen (human in the loop) werden dadurch nicht irrelevant, sondern als ein Aspekt des organisierten Einbettens algorithmischen Bewertens in Entscheidungsarchitekturen sichtbar.

Die beschriebene organisierte Relevantmachung des Algorithmus dadurch, dass er früh in Entscheidungsketten eingebunden ist, lässt sich auch bei notfallmedizinischen Triagierungen und der Eingruppierung von Meldungen als Kinderschutzfälle beobachten. Sie zielen ebenfalls darauf ab, knappe Organisationsleistungen (Aufmerksamkeit, Kompetenz, Zeit) in Relation zu den verfügbaren Ressourcen möglichst effizient zu verteilen. So werden die Kategorien der Triage erst dadurch relevant, dass sie zu Beginn der organisierten Bearbeitung vergeben und nur dann aktualisiert oder verändert werden, wenn die vorhandenen Ressourcen dies erlauben. Auf ähnliche Weise verleihen Jugendämter durch sogenannte Vernotfallung der Kategorie des Kinderschutzfalls im Tagesgeschäft der Fallbearbeitung besondere Prominenz (Büchner 2018b, S. 116). Werden Meldungen als Kinderschutzfall klassifiziert, entsteht eine neue Dringlichkeit der Bearbeitung; zugleich sind situativ mehr personelle Ressourcen gefordert, da Kinderschutzfälle es erfordern, eine weitere Fachkraft hinzuzuziehen. In beiden Fällen etablieren Organisationen Masterkategorien durch Priorisierung und einseitig standardisierte Kategorisierung. Im ersten Fall, indem die angeführten Kategorien nur in Ausnahmefällen bei Großschadenslagen eingesetzt werden, im zweiten Fall, indem Kategorien fallbezogene Ausnahmen vom Regelbetrieb der Fallbearbeitung markieren. 


\subsubsection{Handlungsrelevanz zwischen Beobachtung, Behauptung und Aushandlung- der Algorithmus als Entscheidungshilfe}

Seitens des AMS-Entwicklungsunternehmens wird die Handlungsrelevanz des Algorithmus dadurch relativiert, dass er lediglich als Entscheidungshilfe fungieren soll. Entsprechend wird hervorgehoben, der Algorithmus diene nicht dazu, arbeitssuchende Personen automatisch zu kategorisieren, sondern vielmehr dazu, die Entscheidungsfindung der Vermittler zu unterstützen (Gamper et al. 2020, S. 4). Die hier beschriebene organisationale Einbettung des Algorithmus lässt seine Rolle als Entscheidungshilfe jedoch zweifelhaft werden. Dies begründet sich zunächst darin, dass der Algorithmus eingeführt wird, um ,nachvollziehbare[r] Standards“ (Gamper et al. 2020, S. 4) zu etablieren. Dies stellt jede Abweichung unter den Verdacht, nicht hinreichend begründet vorgenommen worden zu sein, und setzt damit Vermittler einem erhöhten Begründungszwang aus (Büchner und Muster 2018). Verstärkt wird diese Tendenz durch die Idee einer - vermeintlich - höheren Objektivität des algorithmischen Urteils, die sowohl übergreifend die Einführung digitaler Systeme begleitet (Elish und boyd 2018) als auch im AMS-Fall die Stellungnahmen aus Leitungspositionen prägt (Szigetvari 2019; Grund-Groiss 2020; Kopf 2018). Nicht zuletzt sind hier aber auch die bereits thematisierten begrenzten Zeit- und Aufmerksamkeitsressourcen der Vermittler zu nennen. Insbesondere in dieser Hinsicht wird deutlich, dass algorithmisch kein Erkenntnisproblem, sondern ein praktisches Problem gelöst wird (Spiegelhalter 2019, S. 145). Den Algorithmus angesichts dieser typischen organisationalen Situiertheit infrage zu stellen, ist entsprechend zunächst unpraktisch. Dass die Vermittler den Algorithmus kontrollieren, ist aber auch unwahrscheinlich, weil solch eine Kontrolle vertieftes Wissen über die algorithmische Funktionsweise wie auch die Datenbasis voraussetzt. Insgesamt lassen es diese Faktoren, die allesamt aus der organisationalen Situiertheit der Vermittlungssituation resultieren, kaum plausibel erscheinen, dass die Handlungsrelevanz des Algorithmus systematisch durch Vermittler begrenzt wird.

Insgesamt hat dieser Abschnitt argumentiert, dass die soziale Wirkmächtigkeit des Algorithmus auf seiner organisationalen Einbettung basiert. Die einzelnen Aspekte dieser organisationalen Einbettung sind die im Verlaufsprozess früh ansetzende Differenzierung von Klienten, die Konditionierung von Unterstützungsleistungen auf dieser Basis sowie die einseitige standardisierte Re-Kategorisierung von Klienten. Diese Wirkmechanismen erweisen sich jedoch ebenso wie die algorithmisch konstruierte Realität als kontingent. So müssten die berechneten Integrationschancen nicht zwangsläufig früh im Prozess der Fallbearbeitung als konditionales Kriterium genutzt werden; der Rückgriff auf sie könnte zu einem späteren Zeitpunkt stattfinden. Solch ein späterer Rückgriff bei identischer algorithmischer Operationsweise im Prozess der Fallbearbeitung würde ihren Folgenreichtum deutlich relativieren. Dies ließe sich auch erreichen, wenn man den Algorithmus nicht konditional in Entscheidungsprozesse einbinden, sondern als ein mögliches Mittel konzeptualisieren würde, das genutzt werden kann, nicht aber genutzt werden muss oder das flexibel und explorativ statt stabil eingebunden wird. 


\section{Funktionieren als Problem - Lernchancen in der Nutzung algorithmischer Entscheidungsunterstützung}

Die prominente Einbindung des Algorithmus in die Entscheidungsarchitektur des AMS führt zur Frage, wie die beschriebene Kopplung zwischen digitalisiertem Beobachtungsformat und organisationaler Entscheidungsfindung analytisch einzuordnen ist. Um dies zu reflektieren, wird im Folgenden gefragt, inwiefern zu erwarten ist, dass der mit großen Effizienz- und Objektivitätsversprechen eingeführte AMSAlgorithmus Hinweise darauf liefern kann, dass er zu inadäquaten Ergebnissen führt, also die Integrationswahrscheinlichkeit arbeitssuchender Personen systematisch unzutreffend bewertet. Damit schließen wir an Forschungen an, die darauf hinweisen, dass Algorithmen dazu tendieren können, sich auch bei inadäquater Funktionsweise zu stabilisieren (O’Neil 2017; Danaher 2016).

Ausgangspunkt der folgenden Überlegungen ist die Differenz zwischen Singleund Double-Loop-Lernen (Argyris 1976). Unter Single-Loop-Lernen verstehen wir, dass der AMS im Rahmen der Prämissen des Algorithmus lernt, wohingegen wir unter Double-Loop-Lernen verstehen, dass die Prämissen des Algorithmus hinterfragt werden. Blickt man mittels dieser Unterscheidung auf den AMS-Algorithmus, lässt sich zunächst ein Single-Loop-Lernen konstatieren, da die Datensätze des Algorithmus wie auch die Gewichtung der Faktoren regelmäßig aktualisiert werden. Auch ignoriert der AMS nicht, dass Personen falsch kategorisiert werden können. Ist dies der Fall, führt die Neubewertung zu festgelegten Zeitpunkten dazu, dass die Person neu eingeordnet wird und damit gegebenenfalls mehr (oder auch weniger) Unterstützung beanspruchen kann (Gamper et al. 2020, S. 61). In dieser Hinsicht verfügt der Algorithmus folglich über einen Korrekturmechanismus, der aus seiner organisationalen Einbindung resultiert; auch wenn die ursprüngliche Fehlkategorisierung zulasten der Person geht, der geringere Unterstützungsleistungen zugestanden worden waren. Zudem ist zu erwarten, dass der AMS als Organisation zunehmend besser lernt (Argote 2013), mit algorithmisch erzeugter Ambiguität umzugehen, etwa indem sich routinierte Praktiken des Umgangs mit Fällen einspielen, in denen Algorithmus und Fallbearbeiter widersprüchlich votieren. Dieses Lernen ist ergebnisoffen, kann also die unkritische Übernahme des Vorschlags ebenso umfassen wie die routinierte Korrektur des Ergebnisses bei bestimmten Fallkonstellationen.

Fraglich ist jedoch, ob der AMS über ein Single-Loop-Lernen hinaus auch ein Double-Loop-Lernen realisieren und damit die Prämissen des AMS infrage stellen kann. Hierzu wäre es notwendig, dass der AMS evaluieren könnte, inwiefern die algorithmische Bewertungen seines Algorithmus zu adäquaten Ergebnissen führen. Gegen diese Möglichkeit sprechen jedoch mehrere Gründe. Zunächst wird der AMS-Algorithmus bereits mit der nicht unerheblichen und akzeptierten Fehlerquote von ca. $20 \%$ eingeführt (vgl. Gamper et al. 2020). Weiterhin wird ein an den Prämissen des AMS-Algorithmus ansetzendes Double-Loop-Learning aber auch unwahrscheinlich, weil der AMS-Algorithmus nicht vergleichend evaluiert wird und deshalb strukturell von potenziellen Gegenbeweisen verschont bleibt. Ein weiterer wichtiger Aspekt ist der oben bereits angesprochene Umstand, dass personenbezogene Organisationen wie der AMS unter den Bedingungen von Koproduktion und Technologiedefizit operieren (Hasenfeld 2010; Klatetzki 2010; Büchner 2018b). 
Die Folge ist, dass strukturell unklar ist, inwieweit erfolgreiche oder misslungene Arbeitsmarktintegration der Leistung der Organisation zugerechnet werden kann (Technologiedefizit). Die Erfolgsbewertung in diesen Organisationen wird auch dadurch erschwert, dass zentrale Aspekte der Leistungen nicht einseitig, sondern nur bei gelingender Zusammenarbeit zwischen Klient und Vermittler, realisiert werden können (Koproduktion). Im Fall von Ermittlungserfolgen besteht damit systematisch die Möglichkeit, dass diese trotz und nicht aufgrund der algorithmischen Kategorisierung und Leistungszuweisung zustande kommen, etwa weil betroffene Personen unzureichende Leistungszuweisungen durch persönliche Netzwerke neutralisieren oder von Aufschwüngen am Arbeitsmarkt profitieren. Das Feedback, das erfolgreiche Integration generiert, ist folglich strukturell ambivalent, da aufseiten der Organisation unbekannt bleibt, ob sich der Erfolg der eigenen Leistungszuweisung oder externen Faktoren verdankt. Da AMS und Algorithmus diese Ambivalenz jedoch nicht berücksichtigen, lässt sich kaum einschätzen, ob der Algorithmus selbst adäquat funktioniert. Ein Double-Loop-Lernen, das die Prämissen des algorithmischen Ermittlungserfolges problematisiert, ist durch dieses ambivalente Feedback unwahrscheinlich. Insgesamt sprechen die genannten Gründe dafür, dass sich der Algorithmus als verlässlich funktionierende Kategorisierungsinstanz auch bei inadäquaten Ergebnissen stabilisiert.

\section{Schluss}

Die vorangegangenen Ausführungen haben gezeigt, auf welche Weise der AMSAlgorithmus als digitales Beobachtungsformat in die Entscheidungsarchitektur des AMS eingebunden ist. Vor diesem Hintergrund fassen wir an dieser Stelle die zentralen Beiträge des vorliegenden Artikels abschließend zusammen.

Der erste Beitrag des vorliegenden Textes liegt darin, zu zeigen, wie der AMSAlgorithmus seine Realität konstruiert. Dies geschieht, indem er Personen anhand vorab festgelegter Kategorien kategorisiert, vergleicht und deren Integrationschancen darauf basierend bewertet. Wir haben dabei betont, dass es sich hierbei um eine Konstruktion handelt, deren primäres Merkmal ist, dass sie für organisationale Entscheidungen anschlussfähig gemacht wird, um praktische Probleme zu lösen, und nicht etwa, um die Welt komplexitätsadäquat abzubilden.

Unser zweiter Beitrag liegt darin, herausgearbeitet zu haben, dass und wie der Algorithmus durch organisationale Einbettungen actionable gemacht wird und dass hierauf seine soziale Wirkmächtigkeit (Beer 2017) basiert. Zunächst haben wir gezeigt, dass der AMS-Algorithmus die bestehende organisationale Entscheidungsarchitektur lediglich punktuell erweitert. Dass der Algorithmus dennoch eine zentrale Rolle spielt, verdankt sich der fixen Einbettung des algorithmischen Outputs in den organisierten Entscheidungsprozess. Damit werden im Unterschied zur flexiblen Einbettung des Algorithmus bei der explorativen Nutzung von Business Analytics keine Überschussmöglichkeiten produziert. Die Prominenz der Kategorisierungsleistung des Algorithmus basiert darüber hinaus darauf, dass die algorithmische Kategorisierung von Klienten früh in den Entscheidungsprozess integriert ist und einseitig zu fixen Zeitpunkten vollzogen wird. Die im Algorithmus angelegten Kategorien, 
Bewertungen und Vergleiche werden dadurch gerade nicht zu sozialen Randphänomenen, sondern bleiben in ihrer oben rekonstruierten Kontingenz, Gerichtetheit und Spezifik sozial bedeutsam.

Unser dritter Beitrag liegt darin, am Fall des AMS gezeigt zu haben, dass und wie sich Organisationen in die Datenkorpora von algorithmischer Entscheidungsunterstützung einschreiben, z. B. über organisationalen Datenpragmatismus. Zudem haben wir beschrieben, wie Prädiktion als Fortschreibung von vergangener Bewertungskontinuität erzeugt wird und wie Kategorienräume der Prädiktion in diesem Prozess abgedichtet werden. Insofern zeigt sich am AMS-Algorithmus auch, dass algorithmische Systeme in ihrer Modellierung starke und widersprüchliche organisationale Selbstbezüge schaffen. Im hier untersuchten Fall wurden die eigenen, oft koproduktiv erzeugten Leistungen als Vermittlungsorganisation zu weiten Teilen aus der Berechnung von Integrationschancen Arbeitssuchender ausgeblendet. Darüber hinaus haben wir am AMS-Algorithmus gezeigt, dass trotz der Hoffnung, dass algorithmische Unterstützung eine personalisiertere Fallbearbeitung ermöglicht, tatsächlich Fallorientierung befördert wird. Diese wiederum individualisiert strukturelle Benachteiligungen, indem sie diese in individuelle Integrationschancen übersetzt.

Unser vierter Beitrag liegt im Aufgriff der wichtigen Anschlussfrage, ob Algorithmen organisationales Lernen befördern oder erschweren. Dies haben wir am AMSFall betrachtet und argumentiert, dass es gute Gründe gibt für die Annahme, dass sich der Algorithmus in diesem Fall gegen negatives Feedback immunisiert. Angesichts der Ubiquität des zugrunde liegenden Verteilungsproblems und der in diesem Fall beobachtbaren prominenten Positionierung des AMS-Algorithmus bei dieser Verteilung ist dies ein wichtiger Aspekt für zukünftige soziologische Forschung.

Insgesamt zeigt die hier eingenommene relationale Perspektive auf das Zusammenspiel von Organisationen und algorithmischen Entscheidungssystemen, dass die soziale Relevanz, die Algorithmen entfalten, davon abhängt, wie sie in organisierte Entscheidungsarchitekturen eingebettet sind. Wir haben gezeigt, dass eine empirisch rein informierend-subsidiäre Nutzung des Algorithmus eher unwahrscheinlich bzw. angesichts der organisationstypischen Situierung voraussetzungsvoll ist. Für weitere Forschungen regt die aufgezeigte Perspektive an, den soziologischen Blick auf algorithmische Beobachtungsformate in Organisationen neu zu justieren, indem die Gegenüberstellung von menschlichem und maschinellem Entscheiden durch die dargelegte relationale Perspektive zu Organisationen und ihren Entscheidungsarchitekturen ergänzt wird. Eine solche relationale Perspektive konstatiert nicht nur, dass algorithmische Systeme und ihre Kategorisierung-, Vergleichs- und Bewertungsprozesse eingebettet sind, sondern benennt, wie diese Einbettung geschieht. Damit eröffnet sie wichtige Differenzierungsmöglichkeiten für die soziologische Analyse hybrider Entscheidungssysteme. Zugleich vermag eine solche relationale Perspektive, Unterscheidungen und Vergleiche unterschiedlicher Typen und Einsatzformen von Algorithmen zu orientieren. Hilfreich ist dabei, dass Algorithmen in ihrer Mehrdimensionalität, also wie hier als Instanzen zur Distribution öffentlicher begrenzter Ressourcen und zur Entscheidungsoptimierung analysiert werden können. Zudem idealisiert diese Perspektive menschliches Entscheiden nicht, sondern berücksichtigt die Vorstrukturierung von Fallbearbeitung durch Organisationen, Grenzstellen und Algorithmen. 
Danksagung Der vorliegende Text hat maßgeblich von den instruktiven Anmerkungen der Herausgeberinnen, Bettina Heintz und Theresa Wobbe, profitiert. Dies gilt auch für die kritischen und konstruktiven Diskussionen während des Workshops an der Humboldt Universität für das vorliegende Sonderheft. $\mathrm{Zu}$ danken ist ebenfalls Katharina Braunsmann, Korbinian Gall und Justus Rahn sowie den Teilnehmerinnen und Teilnehmern des Kolloquiums am Lehrstuhl für Organisations- und Verwaltungssoziologie der Universität Potsdam für wertvolle Hinweise und Anmerkungen.

Funding Open Access funding enabled and organized by Projekt DEAL.

Open Access Dieser Artikel wird unter der Creative Commons Namensnennung 4.0 International Lizenz veröffentlicht, welche die Nutzung, Vervielfältigung, Bearbeitung, Verbreitung und Wiedergabe in jeglichem Medium und Format erlaubt, sofern Sie den/die ursprünglichen Autor(en) und die Quelle ordnungsgemäß nennen, einen Link zur Creative Commons Lizenz beifügen und angeben, ob Änderungen vorgenommen wurden.

Die in diesem Artikel enthaltenen Bilder und sonstiges Drittmaterial unterliegen ebenfalls der genannten Creative Commons Lizenz, sofern sich aus der Abbildungslegende nichts anderes ergibt. Sofern das betreffende Material nicht unter der genannten Creative Commons Lizenz steht und die betreffende Handlung nicht nach gesetzlichen Vorschriften erlaubt ist, ist für die oben aufgeführten Weiterverwendungen des Materials die Einwilligung des jeweiligen Rechteinhabers einzuholen.

Weitere Details zur Lizenz entnehmen Sie bitte der Lizenzinformation auf http://creativecommons.org/ licenses/by/4.0/deed.de.

\section{Literatur}

Abbott, Andrew. 2014. The Problem of Excess. Sociological Theory 32:1-26.

Amoore, Louise, und Volha Piotukh. 2015. Life beyond big Data. Governing with little Analytics. Economy and Society 44:341-366.

Apelt, Maja und Veronika Tacke (Hrsg.). 2012. Handbuch Organisationstypen. Wiesbaden: VS Verlag.

Argote, Linda. 2013. Organizational Learning. Creating, Retaining and Transferring Knowledge. 2. Aufl. New York: Springer.

Argyris, Chris. 1976. Single-Loop and Double-Loop Models in Research on Decision Making. Administrative Science Quarterly 21:363-375.

Barbour, Joshua B., Jeffrey W. Treem und Brad Kolar. 2018. Analytics and Expert Collaboration. How Individuals Navigate Relationships When Working With Organizational Data. Human Relations 71:256-284.

Bastin, Gilles, und Paola Tubaro. 2018. Le moment big data des Sciences Sociales. Revue française de Sociologie 59:375-394.

Beer, David. 2017. The Social Power of Algorithms. Information, Communication \& Society 20:1-13.

Barnard, Alex V., und Marion Fourcade. 2021. Das Unbehagen an der Ordinalisierung. In Soziale Praktiken des Beobachtens. Vergleichen, Bewerten, Kategorisieren und Quantifizieren, Hrsg. Bettina Heintz und Theresa Wobbe. Sonderheft Kölner Zeitschrift für Soziologie und Sozialpsychologie, (73) (in Druck).

Boelaert, Julien, und Étienne Ollion. 2018. The Great Regression. Machine Learning, Econometrics, and the Future of Quantitative Social Sciences. Revue française de Sociologie 59:475-506.

Brankovic, Jelena, Leopold Ringel und Tobias Werron. 2018. How Rankings Produce Competition: The Case of Global University Rankings. Zeitschrift für Soziologie 47:270-288.

Braudel, Fernand. 1958. Histoire et Sciences Sociales. La longue durée. Annales. Economies, sociétés, civilisations 13: 725-753.

Brayne, Sarah. 2021. Überwachung durch Big Data - das Beispiel der Polizei. In Soziale Praktiken des Beobachtens. Vergleichen, Bewerten, Kategorisieren und Quantifizieren, Hrsg. Bettina Heintz und Theresa Wobbe. Sonderheft Kölner Zeitschrift für Soziologie und Sozialpsychologie, (73) (in Druck).

Büchner, Stefanie. 2018a. Zum Verhältnis von Digitalisierung und Organisation. Zeitschrift für Soziologie 47:332-348.

Büchner, Stefanie. 2018b. Der organisierte Fall. Zur Strukturierung von Fallbearbeitung durch Organisation. Wiesbaden: Springer. 
Büchner, Stefanie, und Judith Muster. 2018. Datafizierung und Organisation. In Datengesellschaft. Einsichten in die Datafizierung des Sozialen, Hrsg. Bianca Prietl und Daniel Houben, 253-270. Bielefeld: Transcript.

Coleman, James S. 1974. Power and the Structure of Society. New York: Norton.

Conrad, Lisa. 2017. Organisation im soziotechnischen Gemenge. Mediale Umschichtungen durch die Einführung von SAP. Bielefeld: transcript.

Danaher, John. 2016. The Threat of Algocracy. Reality, Resistance and Accommodation. Philosophy \& Technology 29:245-268.

Desrosières, Alain. 2001. How Real Are Statistics. Four Possible Attitudes. Social Research 68:339-355.

Elish, Madeleine C., und Danah Boyd. 2018. Situating Methods in the Magic of Big Data and AI. Communication Monographs 85:57-80.

Espeland, Wendy N., und Michael Sauder. 2007. Rankings and Reactivity. How Public Measures Recreate Social Worlds. American Journal of Sociology 113:1-40.

Esposito, Elena, und David Stark. 2019. What's Observed in a Rating? Rankings as Orientation in the Face of Uncertainty. Theory, Culture \& Society 36:3-26.

Fanta, Alexander. 2018. Österreichs Jobcenter richten künftig mit Hilfe von Software über Arbeitslose. Netzpolitik.Org 2018.

Festinger, Leon. 1954. A Theory of Social Comparison Processes. Human Relations 7:117-140.

Fourcade, Marion. 2018. La Logique de la Note. Les Categories morales clans l'Ordre Economique. In Le Désordre du Monde. Rencontres Recherche et Création du Festival d'Avignon, 279-289. Paris: CNRS éditions.

Fourcade, Marion, und Kieran Healy. 2013. Classification Situations. Life-Chances in the Neoliberal Era. Accounting, Organizations and Society 38:559-572.

Frickel, Scott. 2014. Absences. Methodological Note about Nothing, in Particular. Social Epistemology 28:86-95.

Gamper, Jutta, Günter Kernbeiß und Michael Wagner-Pinter. 2020. Das Assistenzsystem AMAS. Zweck, Grundlagen, Anwendung. Arbeitsmarktservice Österreich, Abt. Arbeitsmarktforschung und Berufsinformation/ABI (Hrsg.). Wien: AMS Österreich.

Gigerenzer, Gerd, und Wolfgang Gaissmaier. 2011. Heuristic Decision Making. Annual review of psycho$\log y$ 62:451-482.

Graeff, Peter, und Nina Baur. 2020. Digital Data, Administrative Data, and Survey Compared. Updating the Classical Toolbox for Assessing Data Quality of Big Data, Exemplified by the Generation of Corruption Data. Historical Social Research 45:244-269.

Grund-Groiss, Georg. 2020. Jedem Arbeitslosen das Seine? Wiener Zeitung 2020.

Hacking, Ian. 2001. The Social Construction of What? Cambridge, Mass: Harvard University Press.

Hagendorff, Thilo, und Katharina Wezel. 2019. 15 Challenges for AI. Or what AI (currently) can't Do. AI \& SOCIETY.

Hasenfeld, Yeheskel (Hrsg). 2010. Human Services as Complex Organizations. Los Angeles: Sage.

Heimer, Carol A. 2001. Cases and Biographies. An Essay on Routinization and the Nature of Comparison. American Sociological Review 27:47-76.

Heintz, Bettina. 2010. Numerische Differenz. Überlegungen zu einer Soziologie des (quantitativen) Vergleichs. Zeitschrift für Soziologie 39:162-181.

Heintz, Bettina. 2016. „Wir leben im Zeitalter der Vergleichung.“ Perspektiven einer Soziologie des Vergleichs. Zeitschrift für Soziologie 45:305-323.

Heintz, Bettina. 2019. Vom Komparativ zum Superlativ. Eine kleine Soziologie der Rangliste. In (Be)Werten. Beiträge zur sozialen Konstruktion von Wertigkeit, Hrsg. Stefan Nicolae et al., 45-80. Wiesbaden: Springer.

Heintz, Bettina. 2021. Wie algorithmische Kategorien, Vergleiche und Bewertungen durch Organisationen relevant gemacht werden. In Soziale Praktiken des Beobachtens. Vergleichen, Bewerten, Kategorisieren und Quantifizieren, Hrsg. Bettina Heintz und Theresa Wobbe. Sonderheft Kölner Zeitschrift für Soziologie und Sozialpsychologie, (73) (in Druck).

Holl, Jürgen, Günter Kernbeiß und Michael Wagner-Pinter. 2018. Das AMS-Arbeitsmarktchancen-Modell. Dokumentation zur Methode. Wien.

Illouz, Eva. 2015. Warum Liebe weh tut. Eine soziologische Erklärung. 3. Aufl. Berlin: Suhrkamp.

Kahneman, Daniel, und Amos Tversky, Hrsg. 2000. Choices, Values and Frames. New York: Cambridge University Press.

Kallinikos, Jannis, Hans Hasselbladh und Attila Marton. 2013. Governing Social Practice. Theory and Society 42:395-421. 
Kette, Sven. 2018. Prognostische Leistungsvergleiche. In Vergleich und Leistung in der funktional differenzierten Gesellschaft, Hrsg. Christopher Dorn und Veronika Tacke, 73-98. Wiesbaden: Springer.

Kette, Sven. 2020. „Computer says no?“ Konsequenzen der Algorithmisierung von Entscheidungsprozessen, Ms. (erscheint in: Soziale Systeme. Zeitschrift für soziologische Theorie, Sonderheft ,Organisation und Digitalisierung ', Hg. von Sven Kette und Veronika Tacke, vorauss. 2022)

Kienzl, Sebastian, und András Szigetvari. 2018. Jobchancen-Berechnung. Testen Sie einen der 96 AMSAlgorithmen. Der Standard 2018.

Kitchin, Rob. 2014. The Data Revolution. Big Data, Open Data, Data Infrastructures and Their Consequences. London: Sage.

Kitchin, Rob. 2017. Thinking critically about and researching Algorithms. Information, Communication \& Society 20:14-29.

Klatetzki, Thomas. 2010. Soziale personenbezogene Dienstleistungsorganisationen. Soziologische Perspektiven. Wiesbaden: VS Verlag.

Kleinberg, Jon, Jens Ludwig, Sendhil Mullainathan und Cass R. Sunstein. 2018. Discrimination in the Age of Algorithms. Journal of Legal Analysis 10:113-174.

Kopf, Johannes. 2018. AMS-Algorithmus. Von der Ansicht zur Einsicht. Der Standard 14. November 2018.

Lipsky, Michael. 1980. Street-level Bureaucracy. Dilemmas of the Individual in Public Services. New York: Russell Sage Foundation.

Luhmann, Niklas. 1988. Erkenntnis als Konstruktion. Bern: Bentelli.

Luhmann, Niklas. 1999. Funktionen und Folgen formaler Organisation, 5. Aufl., Berlin: Duncker \& Humblot.

Luhmann, Niklas. 2005. Das Erkenntnisprogramm des Konstruktivismus und die unbekannt bleibende Realität. In Soziologische Aufklärung 5. Konstruktivistische Perspektiven, Hrsg. Niklas Luhmann, 31-57. Wiesbaden: VS Verlag.

Luhmann, Niklas. 2006. Organisation und Entscheidung. 2. Aufl. Wiesbaden: VS Verlag.

MacKenzie, Donald A. 2011. The Credit Crisis as a Problem in the Sociology of Knowledge. American Journal of Sociology Vol. 116:1778-1841.

Maiers, Claire. 2017. Analytics in Action. Users and Predictive Data in the Neonatal Intensive Care Unit. Information, Communication \& Society 20:915-929.

March, James G., und Herbert A. Simon. 1958. Organizations. With the collaboration of Harold Guetzkow. New York u. a.: John Wiley \& Sons.

McCallum, Q. Ethan. 2013. Bad Data Handbook. Mapping the World of Data Problems. Beijing: O'Reilly.

Munn, Luke. 2018. Ferocious Logics. Unmaking the Algorithm. Lüneburg: MESON Press EG.

Noelle-Neumann, Elisabeth. 1974. The Spiral of Silence. A Theory of Public Opinion. Journal of Communication 24:43-51.

O'Neil, Cathy. 2017. Weapons of Math Destruction. How Big Data Increases Inequality and threatens Democracy. New York: B/D/W/Y Broadway Books.

Perrow, Charles. 1991. A Society of Organizations. Theory and Society 20:725-762.

Petersen, Anette C. M., Lars R. Christensen und Thomas T. Hildebrandt. 2020. The Role of Discretion in the Age of Automation. Computer Supported Cooperative Work (CSCW).

Peetz, Thomas. 2021. Digitalisierte intime Bewertung. Möglichkeiten sozialer Beobachtung auf Tinder. In Soziale Praktiken des Beobachtens. Vergleichen, Bewerten, Kategorisieren und Quantifizieren, Hrsg. Bettina Heintz und Theresa Wobbe. Sonderheft Kölner Zeitschrift für Soziologie und Sozialpsychologie, (73) (in Druck).

Pollock, Neil, und Luciana D'Adderio. 2012. Give me a two-by-two Matrix and I will create the Market. Rankings, graphic Visualisations and Sociomateriality. Accounting, Organizations and Society 37:565-586.

Pollock, Neil, und Robin Williams. 2008. Software and Organisations. The Biography of the EnterpriseWide System or How SAP Conquered the World. London: Routledge.

Renard, Léa, und Theresa Wobbe. 2019. La statistique internationale comme instrument de globalisation? La carrière de la catégorie « travailleurs familiaux » au sein de l'Organisation internationale du travail (1919-1982). Revue française de Sociologie 60:595-619.

Schwarting, Rena. 2015. Hochfrequenzhandel zwischen Entscheidungsautomation und Entscheidungsautonomie. In Organisation und Unsicherheit, Hrsg. Maja Apelt und Konstanze Senge, 159-174. Wiesbaden: Springer.

Schwarting, Rena. 2020. Organisation und Verfahren. Zum Veranstaltungsproblem von Verfahren. Soziale Systeme 22:381-423. 
Seaver, Nick. 2017. Algorithms as Culture. Some Tactics for the Ethnography of Algorithmic Systems. Big Data \& Society 4:1-12.

Spiegelhalter, David J. 2019. The Art of Statistics. Learning from Data. London: Penguin Books.

Strulik, Torsten. 2004. Nichtwissen und Vertrauen in der Wissensökonomie. Frankfurt: Campus.

Szigetvari, András. 2019. Arbeitsmarktservice gibt grünes Licht für Algorithmus. Der Standard 2019.

Unternäherer, Markus. 2021. Die Ordnung der Empfehlung. In Soziale Praktiken des Beobachtens. Vergleichen, Bewerten, Kategorisieren und Quantifizieren, Hrsg. Bettina Heintz und Theresa Wobbe. Sonderheft Kölner Zeitschrift für Soziologie und Sozialpsychologie, (73) (in Druck).

Walz, Steffen P., und Sebastian Deterding. 2015. The Gameful World. Approaches, Issues, Applications. Cambridge: MIT Press.

Weinbach, Christine. 2014. Moralische Personenkategorien als Transformationsmechanismus in politischen Dienstleistungsbeziehungen. Das Beispiel der Jobcenter-Interaktion. Zeitschrift für Soziologie 43:150-166.

Wimmer, Barbara. 2020. „AMS-Algorithmus sollte ganz abgedreht werden“. Der Standard.

Wobbe, Theresa. 2012. Making up People. Berufsstatistische Klassifikation, geschlechtliche Kategorisierung und wirtschaftliche Inklusion um 1900 in Deutschland. Zeitschrift für Soziologie 41:41-57.

Wolff, Stephan, und Wolfgang Bonß. 1979. Die Verwaltung sozialer Probleme. Arbeitsbedingungen und Organisationsstrategien der Sozialverwaltung. In Arbeitssituationen in der öffentlichen Verwaltung, Hrsg. Stephan Wolff, 61-114. Frankfurt a. M.: Campus.

Zerubavel, Eviatar. 1996. Lumping and Splitting. Notes on Social Classification. Sociological Forum 11:421-433.

Stefanie Büchner 1982, Dr. rer. pol., Leibniz Universität Hannover, Juniorprofessorin am Arbeitsbereich Soziologie der Digitalisierung, Leiterin der Freigeist Forschungsgruppe Digital Cases. Forschungsschwerpunkte: Wechselverhältnisse von Digitalisierung und Organisation, Fälle und Fallbearbeitung, Organisationssoziologie. Veröffentlichungen: Zum Verhältnis von Digitalisierung und Organisation, Zeitschrift für Soziologie 47, 2018; Der organisierte Fall. Zur Strukturierung von Fallbearbeitung durch Organisation. Wiesbaden 2018.

Henrik Dosdall 1985, Dr. phil., Universität Potsdam, Lehrstuhl für Organisations- und Verwaltungssoziologie. Forschungsschwerpunkte: Entscheidungs- und Organisationstheorie, Veröffentlichungen: Polizeiforschung. Organisationsversagen und NSU-Ermittlungen. Braune-Armee-Fraktion, Behördenlernen und organisationale Suche, Zeitschrift für Soziologie 47, 2018; Die NSU-Ermittlungen, 1998-2011. Eine organisationssoziologische Perspektive, Wiesbaden 2021. 